\title{
Avaliação do processamento auditivo na Neurofibromatose tipo 1
}

\section{Auditory processing evaluation in Neurofibromatosis type 1}

\author{
Pollyanna Barros Batista ${ }^{1}$, Carla Menezes da Silva ${ }^{2}$, Hellen de Oliveira Valentim ${ }^{3}$, \\ Luiz Oswaldo Carneiro Rodrigues ${ }^{4}$, Nilton Alves de Rezende ${ }^{5}$
}

\begin{abstract}
RESUMO
Este trabalho teve como objetivo apresentar os resultados obtidos na avaliação do processamento auditivo de um paciente com Neurofibromatose tipo 1. Embora a audição periférica estivesse normal nos testes realizados, foram observadas alterações importantes no processamento auditivo em várias habilidades. Este achado, descrito pela primeira vez na neurofibromatose, pode contribuir para explicar os distúrbios cognitivos e da aprendizagem já amplamente descritos nesta enfermidade genética comum.
\end{abstract}

Descritores: Neurofibromatose 1; Transtornos da audição; Testes auditivos; Percepção auditiva; Transtornos de aprendizagem

\section{INTRODUÇÃO}

A Neurofibromatose Tipo 1 (NF1) é uma desordem genética autossômica dominante, com penetrância completa que acomete ambos os gêneros, cujas principais características clínicas são as manchas café-com-leite, os neurofibromas cutâneos e plexiformes, as efélides axilares e/ou inguinais e os nódulos de $\operatorname{Lisch}^{(1)}$. A doença é herdada de um dos pais em aproximadamente $50 \%$ dos casos e os demais pacientes não apresentam história familiar de NF1, sugerindo uma alta incidência de novas mutações, que podem ocorrer no óvulo, no espermatozoide ou no embrião(2).

Existe na NF1 alta frequência de problemas psicológicos e de comportamento, além de dificuldades de aprendizagem que resultam em baixo desempenho escolar. Para que haja

Trabalho realizado no Centro de Referência em Neurofibromatose de Minas Gerais e no Ambulatório de Fonoaudiologia do Hospital das Clínicas da Universidade Federal de Minas Gerais - UFMG - Belo Horizonte (MG), Brasil. (1) Pós-graduanda (Mestrado) em Ciências Aplicadas à Saúde do Adulto pela Universidade Federal de Minas Gerais - UFMG - Belo Horizonte (MG), Brasil.

(2) Pós-graduanda (Doutorado) em Ciências Aplicadas à Saúde do Adulto pela Universidade Federal de Minas Gerais - UFMG - Belo Horizonte (MG), Brasil; Professora do Curso de Fonoaudiologia da Pontifícia Universidade Católica de Minas Gerais - PUC-Minas - Belo Horizonte (MG), Brasil.

(3) Mestre em Linguística pela Universidade Federal de Minas Gerais - UFMG

- Belo Horizonte (MG), Brasil.

(4) Doutor, Professor Titular da Escola de Educação Física, Fisioterapia e Terapia Ocupacional da Universidade Federal de Minas Gerais - UFMG Belo Horizonte (MG), Brasil.

(5) Doutor, Professor Associado da Universidade Federal de Minas Gerais UFMG - Belo Horizonte (MG), Brasil.

Endereço para correspondência: Nilton Alves de Rezende. R. Aimorés, 462/116, Funcionários, Belo Horizonte (MG), Brasil, CEP: 30140-070.

E-mail: narezende@terra.com.br.

Recebido em: 15/12/2009; Aceito em: 28/9/2010 aprendizado é necessário que o indivíduo seja capaz de escutar, compreender e separar as partes relevantes da fala dos ruídos de fundo, em qualquer ambiente físico ${ }^{(3)}$.

$\mathrm{O}$ processamento auditivo (PA) refere-se aos mecanismos e processos do sistema auditivo responsáveis pelos seguintes fenômenos comportamentais: localização sonora e lateralização, discriminação auditiva, reconhecimento do padrão auditivo, aspectos temporais da audição, desempenho auditivo com sinais acústicos degradados e desempenho auditivo com sinais acústicos competitivos ${ }^{(4)}$. A desordem do processamento auditivo (DPA) é um distúrbio caracterizado por disfunção em um ou mais mecanismos e processos auditivos anteriormente citados que envolvem estruturas do tronco encefálico, corpo caloso, lobos temporais, parietais, frontais e occipitais e dos processos cognitivos (memória, atenção e linguagem) que interfiram nas análises dos códigos sensoriais auditivos ${ }^{(5)}$.

$\mathrm{O}$ presente trabalho tem como objetivo avaliar e caracterizar o processamento auditivo de um paciente com NF1, atendido pela equipe interdisciplinar do Centro de Referência em NF do HC-UFMG, em testes comportamentais com estímulos verbais e não verbais, e correlacionar os achados destas avaliações com os resultados da avaliação de linguagem, com o intuito de descrever considerações acerca dos distúrbios de aprendizagem frequentemente encontrados na NF1.

\section{APRESENTAÇÃO DO CASO CLÍNICO}

\section{Descrição do sujeito}

Paciente LSV, masculino, trinta e um anos, ensino médio completo, destro, com diagnóstico de NF1 aos quinze anos e único de seis irmãos que apresenta a doença. Foi avaliado no Centro de Referência em Neurofibromatose do 
Hospital das Clínicas da Universidade Federal de Minas Gerais (CRNF) por apresentar incômodo estético com os neurofibromas cutâneos, dor em alguns deles e dificuldade de aprendizagem que iniciaram na infância, principalmente após fracasso escolar na $3^{\mathrm{a}}$ e $5^{\mathrm{a}}$ séries do ensino fundamental. Após procedimentos clínicos e diante do resultado normal do exame de ressonância magnética do encéfalo, o paciente foi encaminhado para avaliação fonoaudiológica como parte rotineira do atendimento do CRNF.

Inicialmente o paciente foi submetido à anamnese, que permitiu o levantamento das seguintes queixas: dificuldade de reter informações na leitura, baixo desempenho na produção escrita, com letra ilegível e rendimento acadêmico insatisfatório, o que culminou com o abandono do curso de economia no primeiro período, além de dificuldades de compreensão de fala em ambientes acusticamente desfavoráveis, seguir conversas longas e grande dificuldade de memorizar músicas.

A avaliação fonoaudiológica compreendeu a avaliação completa do processamento auditivo (audiometria tonal liminar, medidas de imitância acústica e processamento auditivo central) e linguagem oral e escrita.

O paciente também passou por avaliação otorrinolaringológica para complementação diagnóstica sem apresentar alterações e assinou o termo de consentimento livre e esclarecido no que se refere à utilização do seu caso para fins científicos, segundo resolução 196/96 da Comissão Nacional de Ética em Pesquisa (CONEP- FR 267157).

\section{Avaliação do processamento auditivo}

O paciente foi submetido inicialmente à avaliação audio- lógica convencional que revelou limiares auditivos dentro dos padrões de normalidade e logoaudiometria compatível com os limiares tonais. A pesquisa dos limiares ocorreu nas frequências sonoras de $250 \mathrm{~Hz}, 500 \mathrm{~Hz}, 1 \mathrm{kHz}, 2 \mathrm{kHz}, 3 \mathrm{kHz}, 4 \mathrm{kHz}, 6$ $\mathrm{kHz}$ e $8 \mathrm{kHz}$, sendo o critério de limiar de audibilidade normal utilizado foi a presença de níveis de audição inferiores a 25 dBNA em todas as frequências sonoras avaliadas.

As medidas de imitância acústica mostraram timpanogramas tipo A com reflexos acústicos presentes bilateralmente, considerados normais aqueles entre 70-90 dB para as frequências sonoras de $500 \mathrm{~Hz}, 1,2$ e $4 \mathrm{kHz}$.

A avaliação do processamento auditivo foi realizada com o equipamento PAC-2002 da marca Acústica Orlandi ${ }^{\circledR}$ acoplado a um CD player, com fones TDH-39 (padrão ANSI, 1969). Foram utilizados nove testes, a saber: teste de localização sonora, teste de sequencialização de sons verbais e não verbais, teste de fala com ruído branco ${ }^{(6)}$, teste padrão de frequência, teste padrão de duração ${ }^{(7)}$, teste dicótico de dígitos, teste dicótico não verbal e teste dicótico de dissílabos alternados $(\mathrm{SSW})^{(6)}$.

Os resultados dos testes dióticos (estímulos iguais são apresentados simultaneamente para ambas as orelhas), testes monóticos (estímulos diferentes são apresentados simultaneamente na mesma orelha) e dos testes dicóticos (estímulos diferentes são apresentados simultaneamente uma para cada orelha) estão representados nas Tabelas 1, 2 e 3 respectivamente.

A avaliação do processamento auditivo revelou alterações gnósicas auditivas, ou seja, dificuldade na gestão do conhecimento via audição, com alterações nas categorias de decodificação, codificação e de processos temporais.

Tabela 1. Resultados dos testes dióticos e os respectivos padrões de normalidade, habilidades auditivas avaliadas e a categorização da desordem do processamento auditivo

\begin{tabular}{lcccc}
\hline Testes dióticos & Acertos & Padrão de normalidade & Habilidade auditiva avaliada & Categorização da DPAC \\
\hline Localização Sonora & 4 & $\geq 4 / 5$ & Localização sonora & Decodificação \\
Sequencialização de sons verbais & 2 & $\geq 2 / 3$ & Ordenação temporal de sons & Organização \\
Sequencialização de sons não verbais & 3 & $\geq 2 / 3$ & Ordenação temporal de sons & Organização \\
\hline
\end{tabular}

Legenda: DPAC = desordem do processamento auditivo central

Tabela 2. Resultados dos testes monóticos e os respectivos padrões de normalidade, habilidades auditivas avaliadas e a categorização da desordem do processamento auditivo

\begin{tabular}{|c|c|c|c|c|c|}
\hline Testes monóticos & & Acertos & $\begin{array}{l}\text { Padrão de } \\
\text { normalidade }\end{array}$ & $\begin{array}{l}\text { Habilidade auditiva } \\
\text { avaliada }\end{array}$ & $\begin{array}{c}\text { Categorização da } \\
\text { DPAC }\end{array}$ \\
\hline Fala com ruído branco & $\mathrm{F} / \mathrm{R}=+5$ & $\begin{array}{l}\mathrm{OD}=68 \% \\
\mathrm{OE}=80 \%\end{array}$ & $\geq 70 \%$ & Fechamento & Decodificação \\
\hline \multirow{2}{*}{$\begin{array}{l}\text { Padrão de frequência } \\
\text { de três sons }\end{array}$} & Murmúrio & $\begin{array}{l}\mathrm{OD}=40 \% \\
\mathrm{OE}=40 \%\end{array}$ & $\geq 70 \%$ & \multirow{2}{*}{$\begin{array}{l}\text { Reconhecimento de } \\
\text { padrão de frequência }\end{array}$} & \multirow{2}{*}{$\begin{array}{c}\text { Alteração dos } \\
\text { processos temporais }\end{array}$} \\
\hline & Nomeação & $\begin{array}{l}O D=20 \% \\
O E=30 \%\end{array}$ & $\geq 70 \%$ & & \\
\hline \multirow{2}{*}{$\begin{array}{l}\text { Padrão de duração de } \\
\text { três sons }\end{array}$} & Murmúrio & $\begin{array}{l}O D=100 \% \\
O E=80 \%\end{array}$ & $\geq 100 \%$ & \multirow{2}{*}{$\begin{array}{l}\text { Reconhecimento de } \\
\text { padrão de duração }\end{array}$} & \multirow{2}{*}{$\begin{array}{c}\text { Alteração dos } \\
\text { processos temporais }\end{array}$} \\
\hline & Nomeação & $\begin{array}{l}\mathrm{OD}=60 \% \\
\mathrm{OE}=90 \%\end{array}$ & $\geq 100 \%$ & & \\
\hline
\end{tabular}

Legenda: $\mathrm{OD}=$ orelha direita; $\mathrm{OE}=$ orelha esquerda; $\mathrm{F} / \mathrm{R}=$ fala/ruído; $\mathrm{DPAC}=$ desordem do processamento auditivo central 
Tabela 3. Resultados dos testes dicóticos e os respectivos padrões de normalidade, habilidades auditivas avaliadas e a categorização da desordem do processamento auditivo

\begin{tabular}{|c|c|c|c|c|c|}
\hline Testes dicóticos & & Acertos & Padrão de normalidade & $\begin{array}{c}\text { Habilidade auditiva } \\
\text { avaliada }\end{array}$ & $\begin{array}{c}\text { Categorização da } \\
\text { DPAC }\end{array}$ \\
\hline Dicótico de dígitos & & $\begin{array}{c}\mathrm{OD}=92,5 \% \\
\mathrm{OE}=95 \%\end{array}$ & $\geq 95 \%$ & Figura fundo & Decodificação \\
\hline \multirow{3}{*}{$\begin{array}{l}\text { Dicótico não } \\
\text { verbal (12 pares) }\end{array}$} & Atenção livre & $\begin{array}{l}\mathrm{OD}=7 \\
\mathrm{OE}=5\end{array}$ & $\mathrm{OD}=\mathrm{OE}=6( \pm 1)$ & & \multirow{3}{*}{$\begin{array}{c}\text { Decodificação não } \\
\text { verbal }\end{array}$} \\
\hline & Atenção à direita & $\begin{array}{l}\mathrm{OD}=9 \\
\mathrm{OE}=3\end{array}$ & $\geq 10$ & Figura fundo & \\
\hline & Atenção à esquerda & $\begin{array}{l}O D=2 \\
O E=10\end{array}$ & $\geq 10$ & & \\
\hline \multirow{4}{*}{ SSW } & $\begin{array}{l}\text { Direita competitiva } \\
\text { Esquerda competitiva }\end{array}$ & $\begin{array}{c}95 \% \\
12,5 \%\end{array}$ & $\geq 90 \%$ & \multirow{4}{*}{ Figura fundo } & \multirow{4}{*}{$\begin{array}{l}\text { Decodificação, } \\
\text { Codificação e } \\
\text { Organização }\end{array}$} \\
\hline & $\begin{array}{l}\text { Inversões } \\
\text { Tipo A }\end{array}$ & $\begin{array}{c}1 \\
17\end{array}$ & $\begin{array}{l}\leq 1 \\
\leq 3\end{array}$ & & \\
\hline & Efeito de ordem & -5 & $-3 a+3$ & & \\
\hline & Efeito auditivo & 1 & $-4 a+4$ & & \\
\hline
\end{tabular}

Legenda: $\mathrm{OD}=$ orelha direita; $\mathrm{OE}=$ orelha esquerda; $\mathrm{SSW}=$ teste dicótico de dissílabos alternados; $\mathrm{DPAC}=$ desordem do processamento auditivo central

\section{Avaliação de linguagem oral e escrita}

A avaliação da linguagem oral e escrita foi realizada por meio da leitura e ditado de palavras e pseudopalavras ${ }^{(8)}$, leitura de textos referenciados para a avaliação ${ }^{(9,10)}$ e prova de escrita a partir de tema proposto ${ }^{(8)}$, analisadas e interpretadas segundo os critérios de normalidade adotados na literatura ${ }^{(8)}$. Estas avaliações revelaram: leitura sem entonação, negligência quanto à pontuação, velocidade diminuída, omissões e substituições, dificuldades de compreensão de texto dissertativo e fábula, sendo necessária a releitura dos textos para a compreensão do conteúdo. Quanto à escrita, observamos dificuldades para escrever palavras com fonemas que têm múltiplas representações gráficas (s/ç, s/sc, s/x, s/z, z/x, m/n, r/l), apoio na oralidade (substituição u/l) e confusão am/ão. Avaliação de consciência fonológica ${ }^{(11)}$ revelou alterações de síntese fonêmica, rima, segmentação fonêmica, exclusão fonêmica e transposição fonêmica. A prova de consciência sintática ${ }^{(12)}$ aponta dificuldades de correção de frases agramaticais e de categorização de palavras em verbal, adjetiva ou substantiva.

\section{DISCUSSÃO}

A NF1 é uma condição hereditária caracterizada por alterações multissistêmicas que usualmente aparecem durante a infância ou a adolescência ${ }^{(1,2)}$. Dificuldades referentes ao aprendizado da leitura e da escrita têm sido frequentemente apontadas nos indivíduos com NF1 $1^{(3)}$. É comum a associação entre dificuldades de aprendizagem e prejuízos para processar a informação auditiva ${ }^{(13)}$.

Com a realização dos exames objetivos e comportamentais auditivos, foi possível, para esse caso apresentado, delinear as habilidades auditivas alteradas e correlacioná-las com as dificuldades de aprendizado. Observamos, na avaliação da função auditiva, resultados adequados para a habilidade auditiva de localização sonora e ordenação temporal dos sons (tarefas dióticas) e dificuldades do PA principalmente nas tarefas de estimulação monótica e dicótica.

O teste fala com ruído é utilizado para demonstrar alterações do processamento auditivo e medir a habilidade de fechamento auditivo. O processo de reconhecimento de fala em situações de ruído envolve inicialmente a percepção de características acústicas isoladas dos sons que formam as palavras e posteriormente utilização de pistas contextuais para reconstrução da mensagem. Para que essa habilidade auditiva seja desempenhada com sucesso, o indivíduo deve apresentar bom processamento temporal e mecanismos top-down (memória, atenção e linguagem) atuando na análise dos códigos sensoriais auditivos ${ }^{(5)}$.

Desta maneira, a dificuldade do paciente em processar os estímulos sonoros da fala na presença de ruído pode ocorrer por falha nas habilidades perceptuais básicas e/ou déficits nos mecanismos de processamento top-down que podem oferecer obstáculos para a manipulação da estrutura fonológica da linguagem e, consequentemente, contribuir para o baixo desempenho na escrita deste paciente ${ }^{(14)}$.

A habilidade de figura-fundo, avaliada principalmente pelos testes dicóticos, é essencial na resolução de tarefas cotidianas. A alteração observada no teste dicótico de dígitos indica uma dificuldade do paciente para integrar as informações fornecidas simultaneamente às duas orelhas, o que pode contribuir para as dificuldades em acompanhar uma situação em que várias pessoas conversam ao mesmo tempo, diferenciação dos sons da fala, acentuação, entonação da palavra e ritmo $^{(5)}$.

Alterações no teste dicótico não-verbal à direita e no teste SSW à esquerda sugerem dificuldades de comunicação interhemisférica, o que é compatível com a ideia de que o processamento auditivo se dá nos dois hemisférios e há a necessidade de funcionamento adequado da comunicação inter-hemisférica 
pelo corpo caloso ${ }^{(5)}$. Além disso, os resultados obtidos no SSW sugerem ineficiência de comunicação entre as áreas auditivas primárias e associativas, o que poderia contribuir para a dificuldade do paciente em armazenar informações com sequências longas e com várias etapas, para compreensão adequada da leitura e para seu baixo desempenho nas avaliações de consciência fonológica e sintática ${ }^{(11,12)}$.

O reconhecimento de padrões temporais (frequência e duração) exerce papel fundamental na percepção da fala e no aprendizado e contribui para a identificação do conteúdo afetivo emocional $^{(4,5)}$.

A integridade de ambos os hemisférios cerebrais é importante para a percepção e nomeação dos padrões temporais, visto que o hemisfério direito (geralmente o não dominante) está relacionado à percepção de parâmetros fundamentais (frequência, tempo e intensidade) e o hemisfério esquerdo (geralmente o dominante) é importante para a nomeação dos padrões temporais. Quando a resposta ao teste de padrão de frequência e/ou duração exige apenas a imitação (murmúrio), há somente a participação do hemisfério direito, porém quando a resposta a esses testes exige a verbalização, a integridade do lobo temporal direito, das vias do corpo caloso e do lobo temporal esquerdo é fundamental para a obtenção das respostas adequadas ao teste ${ }^{(15)}$.

As dificuldades encontradas nos resultados da avaliação do PA deste paciente em tarefas que exigiram transferência interhemisférica acarretaram déficit na nomeação de padrões temporais, com melhor desempenho em tarefa de murmúrio, visto que esta não envolve associação com o hemisfério esquerdo responsável pela linguagem. Alterações no processamento temporal refletem dificuldades para esse paciente em compreensão de mensagem com duplo sentido, associar fonema-grafema (s/ç, s/sc, s/x, s/z, z/x, m/n, r/l), apoio na oralidade (substituição $\mathrm{u} / \mathrm{l}$ ), leitura oral com velocidade diminuída e dificuldades de entonação (desrespeito as regras de pontuação) $)^{(5)}$.
Essas dificuldades na resolução dos testes monóticos e dicóticos são compatíveis com as dificuldades do paciente em adquirir ou armazenar informações que se sucedem no tempo. Estratégias compensatórias (pistas visuais, contextuais e linguísticas) podem ter sido utilizadas durante os períodos iniciais de aprendizagem e contribuído para que o paciente alcançasse o término do ensino médio e início do ensino superior. Porém, devido à considerável quantidade de trabalho intelectual exigido principalmente em atividades de leitura, compreensão e expressão de conteúdos complexos no ensino superior, associado às alterações de processamento auditivo e de linguagem existentes, estes fatores podem ter contribuído para o baixo desempenho acadêmico do paciente e abandono do curso superior.

\section{COMENTÁRIOS FINAIS}

Embora este trabalho envolva a descrição da avaliação do processamento auditivo e linguagem em um único paciente, ele sinaliza uma anormalidade a ser mais bem estudada nos pacientes com NF1. A DPA pode estar associada ao acometimento multissistêmico e às características clínicas polimorfas e fenótipos variados da NF1 decorrentes da deficiência da produção de neurofibromina no sistema nervoso. A DPA pode contribuir para os distúrbios da aprendizagem nesta doença, por isso a necessidade de ampliar as investigações e os conhecimentos em busca de maior precisão no diagnóstico das alterações de linguagem e audição, ampliando assim os recursos terapêuticos a fim de melhorar a qualidade de vida dos pacientes com NF1.

\section{AGRADECIMENTOS}

À Fundação de Amparo a Pesquisa de Minas Gerais (FAPEMIG), pelo apoio financeiro concedido (Projeto Pesquizador Mineiro II - PPM-00047-08).

\begin{abstract}
The aim of this study was to present the results obtained in the auditory processing evaluation of a patient with neurofibromatosis type 1. Although the patient presented normal peripheral hearing, auditory processing deficits were identified in several abilities. This finding, described for the first time in neurofibromatosis, might help to explain the cognitive and learning disabilities broadly described for this common genetic disorder.
\end{abstract}

Keywords: Neurofibromatosis 1; Hearing disorders; Hearing tests; Auditory perception; Learning disorders

\title{
REFERÊNCIAS
}

1. Ferner RE, Huson SM, Thomas N, Moss C, Willshaw H, Evans DG, et al. Guidelines for the diagnosis and management of individuals with neurofibromatosis 1. J Med Genet. 2007;44(2):81-8.

2. Friedman JM. Epidemiology of neurofibromatosis type 1. Am J Med Genet. 1999;89(1):1-6. Review.

3. Sousa JF, Toledo LL, Ferreira MCM, Rodrigues LOC, Rezende NA. Neurofibromatose tipo 1: mais comum e grave do que se imagina. Rev Assoc Med Bras (1992). 2009;55(4):394-9.
4. American Speech-Language-Hearing Association. (2005) [Internet]. (Central) Auditory Processing Disorders [Technical Report]. [cited 2010 Mar 12] Available from: http://www.asha.org/docs/pdf/TR2005-00043.pdf.

5. Bellis TJ. Assessment and management of central auditory processing disorders in the educational setting: from science to practice. 2nd ed. New York: Thomson Delmar Learning; 2003.

6. Pereira LD, Schochat E. Processamento auditivo central: manual de avaliação. São Paulo: Lovise; 1997. 
7. Lizarro MBT. Processos temporais auditivos em músico de Petrópolis [monografia]. São Paulo: Universidade Federal de São Paulo. Escola Paulista de Medicina; 1999. $76 \mathrm{f}$

8. Braz HA, Pellicciotti TH. Exame de linguagem TIPITI. São Paulo: MNJ; 1988.

9. Scliar M. Comendo papel. In: Scliar M. Contos reunidos. São Paulo: Companhia das Letras; 1995. p. 41-2.

10. Da Vinci L. A borboleta e a chama da vela. In: Carvalho BB, Camargo L, Machado M. Fábulas e alegorias. São Paulo: Berlendis e Verteccia Editores, 2009, p.17.

11. Santos MTM, Pereira LD.Teste de consciência fonológica. In: Pereira LD, Schochat E. Processamento auditivo central: manual de avaliação. São Paulo: Lovise; 1997. p. 187-98.
12. Capovilla AGS, Capovilla FC. Avaliando a habilidade metassintática por meio da prova de consciência sintática. In: Capovilla FC, Capovilla AGS. Prova de consciência sintática (PCS): normatizada e validada: para avaliar a habilidade metassintática de escolares de $1^{\mathrm{a}}$ a $4^{\mathrm{a}}$ séries do ensino fundamental. São Paulo: Memnon; 2006.

13. Frota S. Processamento auditivo: estudo em crianças com transtornos específicos da leitura e da escrita [tese]. São Paulo: Universidade Federal de São Paulo. Escola Paulista de Medicina; 2003.

14. Idiazábal-Aletxa MA, Saperas-Rodríguez M. Procesamiento auditivo en el trastorno específico del lenguaje. Rev Neurol. 2008;46(Supl 1):S91-5.

15. Musiek FE, Pinheiro ML. Frequency patterns in cochlear, brainstem and cerebral lesions. Audiology. 1987;26(2):76-88. 